\title{
Reliable File Sharing Scheme for Mobile Peer-to-Peer Users Using Epidemic Selective Caching
}

\author{
Constandinos X. Mavromoustakis and Helen D. Karatza \\ Department of Informatics, \\ Aristotle University of Thessaloniki, \\ 54124 Thessaloniki, GREECE \\ \{cmavrom,karatza\}@csd.auth.gr
}

\begin{abstract}
In the recent years, mobility is gaining popularity for non critical civilian applications. Hybrid and pervasive computing environments with billions of users, devices, and services, demand ensured end to end reliability and integrity. However due to the asymmetry in wireless communication and the scarceness of wireless resources, the use of such networks as a mean of file sharing is not always successful. This paper proposes an approach for reliable file sharing based on the advantages of epidemic selective dissemination through fixed and mobile Infostations. The proposed scheme attempts to fill the gap between mobility and reliable file sharing for mobile peer to peer users. Facing the problem of flooding this method successfully enables end to end reliability by forwarding requested packets to epidemically 'selected' users in the network. Thorough examination is made for the performance and reliability of the epidemic selective caching method proving it's robustness in reliable file sharing among mobile peers.
\end{abstract}

\section{Introduction}

As the digital age continues, the role of wireless technology in data telecommunications as well as multimedia applications becomes more apparent with its improved data throughput rate. Different network configurations like MANets, Mobile peer to peer systems (MP2P) or hybrid systems (Infostations [1]) take advantages of user's mobility to improve connectivity, information sharing and sensor characteristics. The nowadays ubiquitous presence of mobile phones and PDAs equipped with close range radio connectivity like Bluetooth and WLAN create a fertile environment for ad hoc networks. These kinds of networks are very flexible and suitable for several situations and applications allowing an infrastructureless network without any preinstalled components. Every node acts as a router and has limited transmission range while the communication traffic has to be relayed over several intermediate nodes (multi-hop) to enable the communication from a source to a destination.

Choosing appropriate information dissemination strategies is crucial in mobile ad hoc networks (MANET) due to the frequent changes in topology. Flooding-based approaches like diffusion have a strong similarity with epidemic spreading of diseases. Applying epidemiological models to information diffusion allows the evaluation of such strategies depending on the MANET characteristics, e.g. the node density. In order to choose appropriate strategies at run time, the model should be easily evaluated. Reliability in highly changing topology networks is a major issue since their moving characteristics trade off the end user's QoS. Thus optimally designed systems have to offer end user reliability and integrity by enabling reliable sharing of information.

Unlike cellular systems where users enjoy a constant connectivity, MP2P systems are "short-lived". Connections between peers on the network are prone to failures. MP2P devices have many negative characteristics like short connections times, unpredictable disconnections (range and battery failure), small network formation factor, and file's unavailability. In this paper a reliable file sharing scheme for MP2P devices is proposed taking the advantages of epidemic file dissemination through fixed and mobile Infostations [1, 9, 10]. Through geographical landscapes where Infostations are set, the modified epidemic protocol creates a replicated object in order to enable MP2P reliable file sharing. This scheme proved its scalability in node's density since it does not require the knowledge of network at any single host. Additionally it does not require spatial 
distributions to efficiently spread information while enables reliability in supported mobility.

The organization of the paper is as follows: Section 2 discusses the related work that has been done for MP2P reliable information sharing, featuring out the basic principles and the conducted solutions by different schemes. Section 3 then introduces the proposed reliable file sharing scheme for MP2P users, followed by section 4 which provides the evaluation and simulation results of the proposed scheme in contrast to the reliability factors for file sharing in MP2P hybrid networks. Finally, Section 5 concludes with a summary of our contribution and further research.

\section{Related work}

MP2P environment represent a new class of computing with large numbers of resource-constrained computing nodes, cooperating on a single or group of applications. MP2P devices must often operate for extended periods of time unattended, where evolving analysis and environments can change application requirements, creating the need to alter the network's behavior. Totally different from the traditional method of programming a node over a dedicated link, the embedded nature of these systems requires a mechanism to propagate new code over the network.

In general, the information sharing process should require a minimal amount of time. This not only reduces any service interruptions to a deployed application, but is also reduces information sharing reliability. However, recent researches in reliable information sharing require dense networks in order to bind network partitioning. However, in dense networks, the contention and collisions caused by the random interactions of neighboring nodes harms the performance since file sharing techniques rely on overhearing. In turn overhearing drives the network to generate a huge overhead in order to "hear" the peers. This issue can be disastrous for sensitive information since capacity limitations can take place in each node due to overhearing, and information will be lost $\mathrm{t}^{\mathrm{t}}$.

For data dissemination in wireless networks, naive retransmission of broadcasts can lead to the broadcast storm problem [2], where redundancy, contention, and collisions impair performance and reliability. The authors in [2] discuss the need to have a controlled retransmission scheme and propose several schemes, such as probabilistic and location based methods. The experiments were conducted by Ganesan et al. In [3]

\footnotetext{
The same time security issues arise.
}

authors identify several interesting effects at the linklayer, notably the highly irregular packet reception contours, the likeliness of asymmetric links, and the complex propagation dynamics of simple protocols. In [4] an epidemic algorithm is proposed based on strictly local interactions for managing replicated databases in a robust way for unpredictable communication failures. The epidemic property is important since MP2P devices experience high loss rates due to their unpredictable movements, their asymmetric connectivity, and to node failures in the "repopulation" process [5]. When a mobile node makes an explicit request for a resource, the whole network is flooded with a query, like mobile ad-hoc route discovery algorithms [6]. The same actions occur for file discovery by query flooding in P2P networks like Gnutella [7]. Significant improvements of the basic flooding approaches using advertisements and geographic information have also been recently studied [8]. This work proposes a reliable autonomous file sharing scheme with use of the new concept of mobile Infostations where is a variant of fixed Infostation proposed in $[1,11,13]$. In this work the file sharing mechanism is essentially stateless, where neither routing information is required nor global information like knowledge of the destination nodes is required. The basic advantage, while enabling high reliability and ensuring that file sharing will complete successfully, is that this scheme does not flood the whole network. By adopting the idea of geographical landscapes where Infostations are set in, the modified epidemic protocol creates a replicated object to mobile Infostations in order to enable P2P reliable file sharing in an "any cast" form.

\section{Reliable File Sharing/Gossiping Scheme for Mobile Peer-to-Peer devices using Epidemic Selective Caching}

This section describes the reliable autonomous file sharing scheme with use of the mobile Infostations, which bounds the dissemination of requested files in geographical landscapes in an epidemic way.

\subsection{Cooperative mobile Infostations in organized landscapes}

Recently, new ideas are emerging contrasting the concept of "run anywhere, anytime" pervasive systems, such as Infostations and ad hoc networks. Such systems are based on the waterfilling concept, where the transmission occurs only when source and destination are close together to ensure reliability. 
Results obtained for these issues $[1,9,11]$ have provided good estimates that permit such systems to become the basis for low cost, wireless data transmission.

The idea of MANETs is also contrasting the ubiquitous coverage paradigm of the cellular systems. But taking into account that the capacity of MANETs is constrained by the mutual interference of concurrent transmissions between nodes, security and reliability issues arise on a mobility based scenario. Some research results presented in [10], introduce mobility into a fixed network model, which show that the average throughput per source-destination pair can be kept constant even if the number of nodes per unit area increases. This improvement is obtained through the exploitation of the time-variation of the user's channel due to mobility. The new concept in [10] is to split the packets of each source node to as many nodes as possible. Therefore, strategies of this type incur additional delay, because packets have to be buffered until the channel becomes sufficiently strong for transmission(s). Taking into account all the above reasons, this paper adopts the idea of Infostation into geographical landscapes. Figure 1 shows the basic Infostation model applied in a geographical landscape.

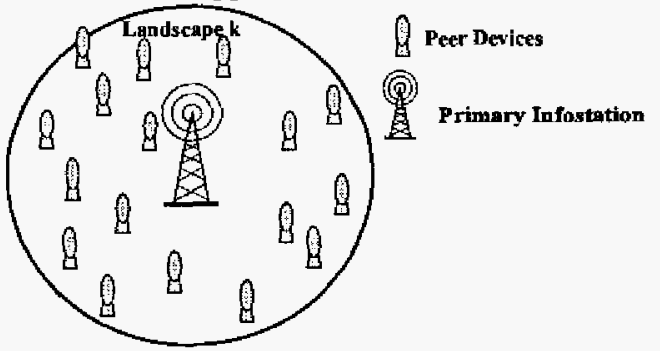

Figure 1: Geographical landscape with the basic Infostation model.

Infostations consist of high bit rate connectivity which can be seen as independent access ports to the Internet, or (clustered) organized into groups having a common server (Cluster Infostation Controller-CIC). Different locations and characteristics of user mobility patterns for different scenarios are depicted in [11, 12]. In such scenarios ${ }^{2}$ the controllers are connected to the internet through a backbone network. Combining the pure Infostation system with a mobility based framework we introduce the Hybrid Infostation System (HIS) where the primary Infostation could be static as well, and having secondary Infostations moving across an area forming a cluster with other peers. This hybrid peer-to-peer architecture resolves the disadvantages of the pure MP2P architecture in terms of inefficient

\footnotetext{
${ }^{2}$ The drive through, the walk-through, and sit-through scenario(stationary users)
}

routing, network partitioning and lack of security. This hybrid Infostation system adopts the basic concept of pure Infostation system in terms of capacity node but without flooding the network with unnecessary flow of information. This capacity node plays a role of control storage node [13]. On the contrary to [13] this work proposes a hybrid peer-to-peer architecture shown figure 2. Fixed Infostation is located in every landscape covering a certain predetermined area. Additionally Mobile Infostations (MIs) are set dynamically. These MIs are users that are in the landscape creating a tree of MIs users and FI users. This principle is shown in figure 2. The coexistence of FI and MIs simultaneously recovers from the split of the peer-to-peer network, and improves the network topology and in the appropriate form the file sharing reliability. In otder to improve the inefficiency of the pure peer-to-peer flooding tactic an epidemic object replication scheme is chosen for teliable file sharing. This scheme is described in the following section.

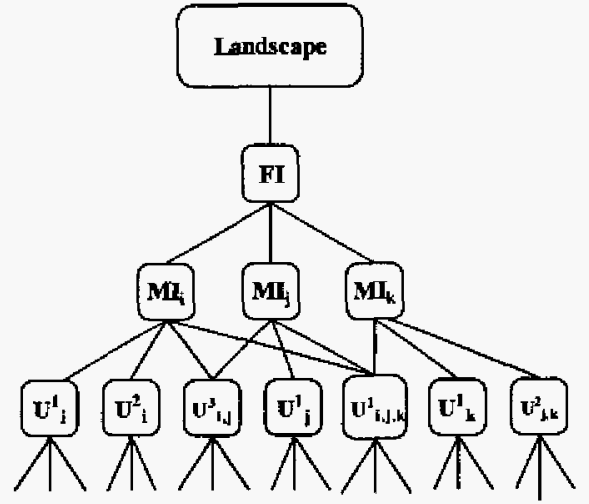

Figure 2: The hybrid peer-to-peer Infostation based architecture.

\subsection{Epidemic object replication scheme for reliable file sharing}

Distributing information within networks can be very complicated particularly if hosts do not have wider knowledge of the properties of the network. As a result many problems occur when it is highly important that a certain information or group of information (file(s)) have to reach one particular host or all hosts within the entire network. Epidemic algorithms follow a nature paradigm by applying simple rules to efficiently spread information by just having a local view of the environment. According to this fact, epidemic algorithms are easy to implement and guarantee message propagation in heterogeneous environments.

To achieve reliability in MP2P environments a dynamic gossiping scheme must be used. Secure file sharing can be determined by relying on epidemic 
algorithms, a breed of distributed algorithms that find inspiration in the theory of epidemics. Epidemic (or gossip) algorithms constitute a scalable, lightweight, and robust way of reliably disseminating information to a recipient or group of recipients, by providing guarantees in probabilistic terms. Based on certain characteristics, epidemic algorithms are amenable to the highly dynamic scenarios. In this work a promiscuous caching is used which means that data can be cached "anywhere, anytime". However this enables trade offs in consistency for availability which is faced with cooperative MI used in landscapes described earlier.

In a MP2P system each user might desire to share or download a file or files with other users (peers). Many conditions must be satisfied for reliable communication between mobile peers. On one hand users due to their mobility might draw away from the user (peer) that a file sharing communication takes place. On the other hand a sudden network partitioning or network split could occur because of network's dynamic topology which is continuously changing. Thus a proactive dissemination scheme must be determined in order to prevent the cutoff in file sharing communication. This work assumes an isolated system comprising of a fixed number of mobile nodes confined in a predefined geographic region. These nodes are mobile, and communicate with each other in a wireless (radio) ad-hoc manner. As studied in $[8,10$, 12] there is a trade off between reliable coverage and data rate. The limited connectivity coverage that MP2P systems offer, results in significant delay in downloading a message or file (group of packets). In the proposed scenario each node carries some unique data items. During the period for which the system is studied, no new node is inserted in the network.

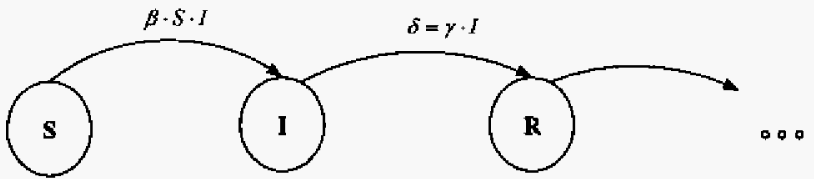

Figure 3: Markov chain model of an infectious disease with susceptible, infected, and recovered states.

Each mobile host $m_{k}$ has a predetermined capacity

$M$. At any time in the network each $m_{k}$ has a state.

There are three states that $m_{k}$ can be characterized: the susceptible state $S(t)$ represents the number of hosts in the system which are "susceptible," infected state $I(t)$ represents the number of "infected" hosts, and $R(t)$ represents the "recovered" hosts. A host is in susceptible state $S(t)$ if the device does not share any information with any other host. In turn $A$ host is in infected state $I(t)$ if a file(s) share occurs. Finally a host is in "recovered" state $R(t)$ if any shared file(s) are no longer pending. A Markov chain model of an infectious disease with susceptible, infected, and recovered states is used shown in figure 3. Markov chain model was chosen to evaluate this extra storage requirement, for modeling of infectious diseases to determine the file sharing termination criteria.

The modelling of diseases in fixed networks have been studied in the past [14], and this model is used in a similar manner in this scenario. Adopting the framework from an infectious disease model [15], a host is set as "infected" if a file sharing (or a group of data packets) are pending. Suppose there are $k$ hosts in the system, then a host is sharing a resource with $\beta(k-1)$ other hosts per unit time. $S(k-1)$ do not have yet the disease. Therefore, the transition rate from state $S$ to state I becomes

$$
\begin{aligned}
& \text { Filesharing }= \\
& (\text { nu_inf ected }) \cdot(\text { dld_Rate }) \cdot\left(n u_{-} N O T_{-} \text {share }\right) \\
& \quad \text { Filesharing }=I[\beta(k-1)] \cdot\left[\frac{S}{k-1}\right]
\end{aligned}
$$

where $\beta$ is the contact rate for $k$ hosts.

Then the downloaded (no longer pending) rate is :

$$
\delta=\gamma \cdot I
$$

where $\gamma$ is the download rate and $l$ is the number of infected devices.

The $\beta \cdot S \cdot I$ is called $\pi$ coefficient which indicates the enforcement degree of the diffusion process. $\pi$ has the dimension of $\left[\frac{1}{\text { Time }}\right]$. Previous examinations of the behaviour of small scale systems [15] showed that relatively small populations could be faced wit a stochastic model. Thus taking into account that $\pi$ depends on the number of $S(t)$ and $I(t)$ and the probability of transmitting the information, we can derive $S(t)$ as follows:

$$
\begin{aligned}
& \frac{d S}{d t}=-\beta \cdot S \cdot I=-\pi \\
& \frac{d I}{d t}=\beta \cdot S \cdot I=\beta(N-I) \cdot I=\beta N I-\beta I^{2}
\end{aligned}
$$

By solving the first order differential equation the outcome is:

$$
I(t)=\frac{N}{1+e^{-\beta \cdot N \cdot}(N-1)}
$$

According to the definition of spreading ratio equation (3.2) becomes: 


$$
\begin{aligned}
& I^{\prime}(t)=\frac{I(t)}{N}=\frac{N}{N \cdot\left(1+e^{-\dot{\beta} \cdot N \cdot 5}(N-1)\right)} \\
& I^{\prime}(t)=\frac{1}{1+e^{-\beta \cdot N \cdot t}(N-1)}
\end{aligned}
$$

Equation 3.3 is referred as the cumulative distribution function.

An issue is when the locations will be updated. This issue can be measured as follows:

$$
L(t)=L(t-1)+S_{1} \cdot \vec{d}
$$

Where $L(t)$ is the new location $L(t-1)$ the previous location at step time $(t-I), S_{t}$ is the speed of each device and $\vec{d}$ is the directed unit vector [13]. Additionally the distance from a node to the closest FI can be measured as [(Node's position - center of FI's communication area) - (radius of area)].

By using the above analytical epidemic approach for packet diffusion the gain is significant as results in the following section show. Epidemic approach with the highly redundant and fault tolerant way is applied in the system only when a packet is disturbed on its way to destination or when sender or receiver notice an unreliable communication. This might be an unpredictable movement, asymmetric connectivity and node failures. There exist two different user-based cases where the communication might be disturbed: (i) when source user's communication fails and (ii) user's destination communication fails. In case (i) the source users might move to a point that no communication coverage exists and as a result connection failure will occur and the prospective resource for download will be lost.

Taking cases (i) and (ii) as a paradigm this paper proposes a solution in order to enable reliability between resource sharing mobile peers as an alternative approach with [23]. Considering a file $^{3}$ download from a node $A$ to a node $B$ it is helpful to evaluate both cases. In (i) the user's device (mobile node) chooses in epidemic form (infection) which of user's device neighbouring nodes, will be MI. This classification is based on candidate's node residual energy, capacity and signal transmission power [22]. FI only communicate with $\mathrm{MI}$ and not with pure users. Node "A" then copies packets to the chosen "infected" MI for time $t$. MI in turn copies these packets to FI of the landscape to which is the only user which communicates directly $[13,21]$. This file lies in FI buffer for time $t$ and then is deleted. This mechanism occurs recursively for the forthcoming chosen MIs, in the case where MI's communication fails. Finally

\footnotetext{
${ }^{3}$ Stream(s) of packets.
}

when downloading is completed, file is being removed from FI and "infected" MI even if time $t$ has not yet ended. Additionally every infected node then recovers.

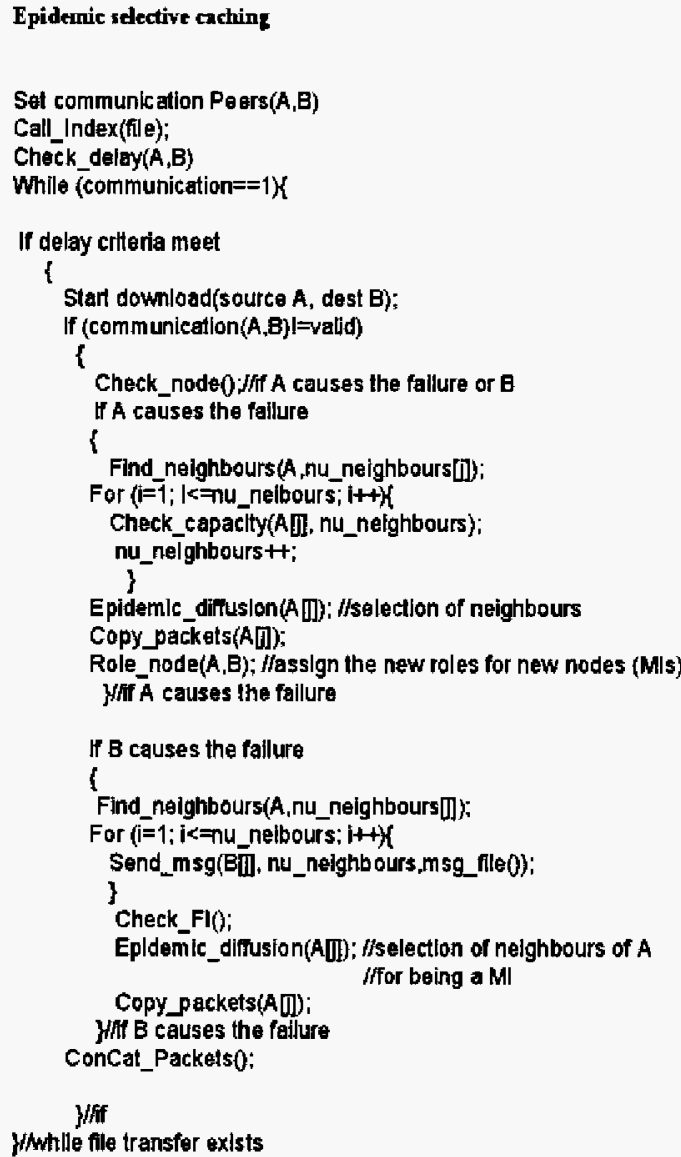

Figure 4: Pseudocode for epidemic selective caching for reliable file sharing scheme between peers.

In case (ii) where the destination node " $B$ " is to be moved influencing the communication between $A$ and $B$, a similar mechanism is activated in the following way: if the signal transmission power is reduced which means communication between $A-B$ is prone to failure(s), "B" sends messages to "A's" neighbours to search for MIs in an epidemic way-as explained earlier. In turn MIs copy packets to FI of the landscape and then FI to MI-cluster controllers to which an examination takes place to examine whether destination node " $\mathrm{B}$ " changed landscape. If " $\mathrm{B}$ " changed landscape then MI-cluster controllers copy file packets to FI of the nearby landscape otherwise the epidemic algorithm of case (i) takes place. These principles are illustrated in the pseudocode of figure 4.

The above method spreads data load evenly in an epidemic way. Assuming that the mobility of hosts is a 
hybridized version of city walk and random walk [16] the epidemic selective method is applied only when any file transfer is disturbed. Each host chooses an anchor randomly as its next target destination, and moves toward the anchor at a variable speed parameterized by average speed. Epidemic packet diffusion enables reliability as shown in the results in the following section.

\section{Simulation experiments and discussion}

The design and evaluation of any heuristic method attempting to solve critical problems like file sharing reliability requires practical understanding of the discrete event mechanisms behavior of the underlying methods. To demonstrate the methodology discussed in this paper, we performed exhaustive discrete time simulations of the proposed scenario under several different conditions.

We assume a system consisting of several mobile nodes, e.g., mobile users equipped with notebooks or PDAs and wireless network interfaces. All mobile nodes collaborate via a shared application that uses a distributed lookup service. Radio coverage is small compared to the area covered by all nodes, so that most nodes cannot contact each other directly. Additionally, we assume IEEE $802.11 \mathrm{x}$ as the underlying radio technology. However, it is necessary to point out that communication and epidemic-like dissemination could be employed on any radio technology that enables broadcast transmissions inside a node's radio coverage.

\subsection{Routing protocol used}

One basic issue is the selection of the routing protocol that should be used in order to cooperate with the described scenario. Considering the need of bandwidth and the limited battery power for wireless devices, it is necessary to apply efficient routing algorithms to create, maintain and repair paths with least possible overhead production [2]. In the described scenario control packets for epidemic selection must actively follow a route in order to transfer certain information. As known there are two classes of routing protocol: proactive and reactive. In proactive or table-driven protocols the routes are maintained for all possible destinations continuouslyperiodically, even if routes will not be actually used. The generated overhead from route maintenance cause significant reduction of network performance, increase in end-to-end delays and delay variations. Reactive or on-demand protocols on the other hand, create and maintain routes only when they are needed.

In the implementation of the proposed scenario the Zone Routing Protocol (ZRP) $[19,20]$ is used. ZRP is a hybrid protocol that combines the reactive and proactive modes. The ZRP is considered advantageous because allows to a certain node to accurately know the neighbors of any mobile terminal within a zone (Infostation-like approach $[1,2,6,19])$. These devices should be in zone that could be accessible in a fixed number of hops. In the proposed scenario zone refers to each nodes connectivity domain as appears in the tree of figure 2. Since ZRP allow the absolute communication with neighbors, is considered less expensive, while neighbors contribute in the routing process. Particularly ZRP divides the network into several routing zones specifying a determined number of hops. This allows the routing protocol to be adjustable for different operational network conditions such as heavy traffic [17-21].

\subsection{Simulation results of the proposed scenario}

To emulate the scenario described earlier, the need of a possible realistic environment must be achieved. In this section, we present some experimental and simulation results for performance evaluation and reliability in resource sharing offered by our scheme.

Two sets of experiments were performed. One set deals with the selective caching concept and the grade of contribution in enabling reliability in file sharing, and the second deals with the performance under significant traffic, network partition limitations and the latency issues that arise. As mentioned the caching capacity of each node could be unlimited while nowadays memory becomes cheaper and cheaper. However if each node has unlimited capacity then enormous traffic will reach each node's buffer causing "vicious" dissemination in a bounded node network. Thus taking into account this issue, this paper evaluates two different types of caching capacity:

(i) Unlimited capacity

(ii) Limited capacity for each node as $64 \mathrm{~KB}, 128 \mathrm{~KB}$, $512 \mathrm{~KB}, 2 \mathrm{MB}, 10 \mathrm{MB}$.

An issue that has to be taken into account is whether the cached information destined for a proper node could be stored in a node with higher residual energy. As shown in simulation process if nodes with higher level of residual energy are chosen in the path then the network partitioning probability is further reduced [21-22]. For this reason cached information size and file size are chosen randomly in our scenario and files are searched for, upon queries on a recursive basis. 
In simulation was used a two-dimensional network, 3 landscapes each one consisting of 25 nodes with each link (frequency channel) having max speed reaching $2 \mathrm{Mb}$ per sec. The propagation path loss is the two-ray model without fading. The network traffic is modeled by generating constant bit rate (CBR) flows. Each source node transmits one 512-bytes ( $\sim$ Kbitslight traffic) packet. Packets generated at every time step by following Pareto distribution as depicted in [17-19], destined for a random destination uniformly selected.

Additionally we have modeled in each node an agent which evaluates the information destined for a proper destination. In this way we have at any time measures of the information destined for each node (for a given time interval) by any node. Network structure has been implemented as a [N-1] row, [N-1] column for each node being a possible destination as developed in [21].

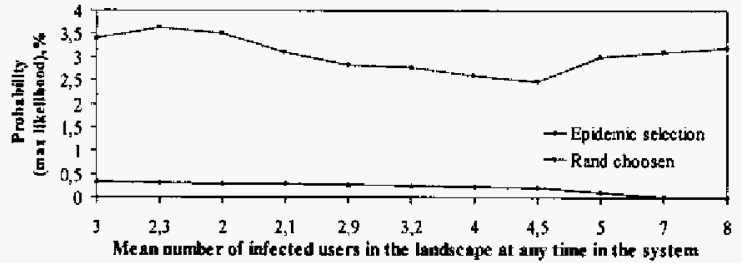

Figure 5: Mean number of infected users in the landscape.

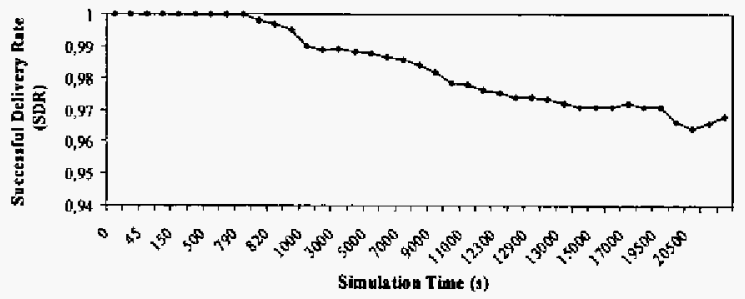

Figure 6: The ratio of successful packet delivery.

Figure 5 illustrates the mean number of infected users, infected by epidemic algorithm used and by randomly chosen selection, in the landscape. As seen the mean number of infected users for which epidemic algorithm is used is significantly small compared with the number of randomly chosen. This proves the robust characteristics in node selection for packet's caching in order to enable reliability.

Figure 6 shows the ratio of successful packet delivery. It is clearly shown that throughout simulation time the SDR drops slightly using the epidemic selection. After consecutive simulations the extracted values did not dropped below $96.5 \%$ for successful delivery of packet. Of course under self similar traffic (web traffic [18]) it is obvious that a trade off of $2,5 \%$ is expected for SDR. Future research will examine such scenario.

Extremely lower than the expected values is the number of transmitted packets versus the packet loss shown in Figure 7. Different traffic measures were examined showing that heavy traffic ( $4^{*}$ light traffic) does not enable better utilization of network resources using epidemic selective caching where packet loss compared with sent packets is high enough. On the contrary moderate and light traffic behave significantly better having less total number of sent packets, lost.

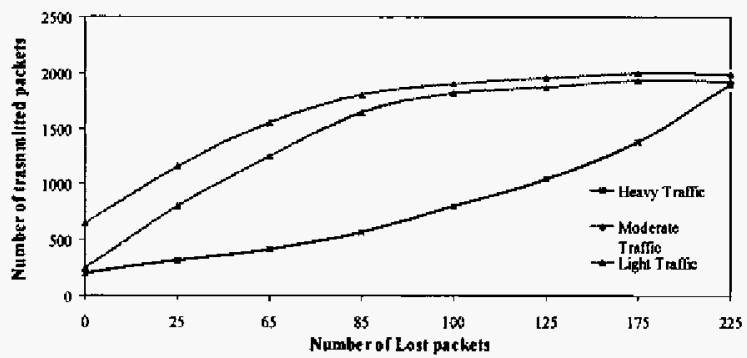

Figure 7: Number of transmitted packets versus the number of lost packets for different traffic volumes.

In figure 8 the average packet delay with the number of injected packets in the network is illustrated. As the number of packets increases the average packet delay increases slightly. After consecutive simulations for this metric it has been shown that when the number of injected packets reaches $550-600$, the average packet delay increases dramatically for $64 \mathrm{~KB}$ capacity. This occurs due to 64KB capacity limitation that binds each node. As shown for different capacity limitations the average delay remain almost the same for $128 \mathrm{~KB}, 512 \mathrm{~KB}, 2$ $\mathrm{MB}$ and $10 \mathrm{MB}$.

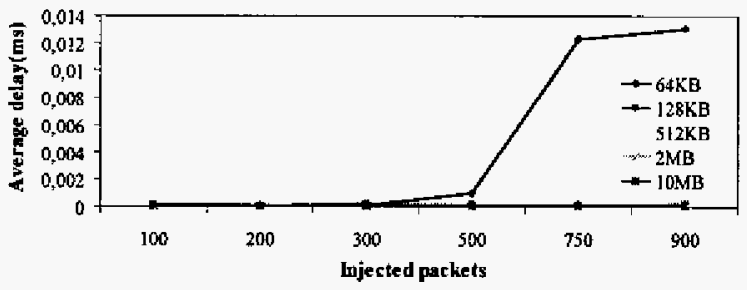

Figure 8: Average packet delay versus the number of injected packets in the network.

Figure 9 shows the percentage of successfully transmitted packets using epidemic selective caching 
versus the mean number of disturbed files. It is remarkable to point out that for large number of file distributions the percentage of successfully transmitted packets using epidemic selective caching does not drops below $89.8 \%$. This means that for large distributions in any landscape the packets can be successfully transmitted in high percentage compared with other schemes $[1,5,11]$.

In figure 10 the average number of segments per file versus the capacity of each file is illustrated. It is easily extracted that for large files (>1MB), the segments which are randomly chosen depending on each file chunks, are not exceeding 64. From figure 10 it is indicated that small files can be segmented (in ratio) in much more pieces than for large files. This results a better reliability response in many consecutive simulation's potshots.

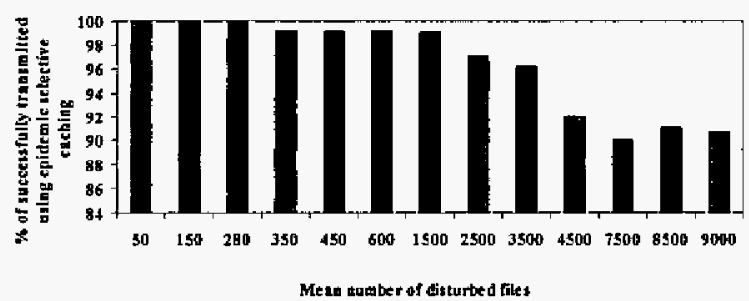

Figure 9: Mean number of disturbed files with the number of successfully transmitted using epidemic selective caching.

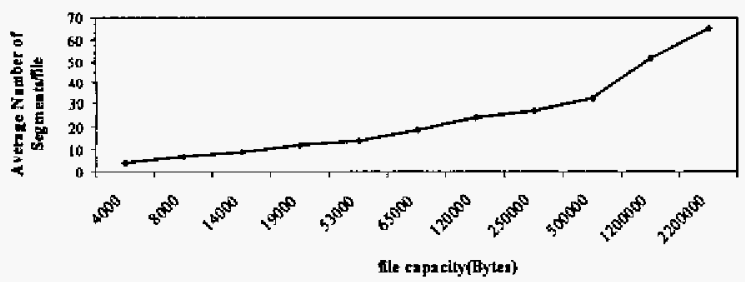

Figure 10: Average number of segments/file versus file capacity.

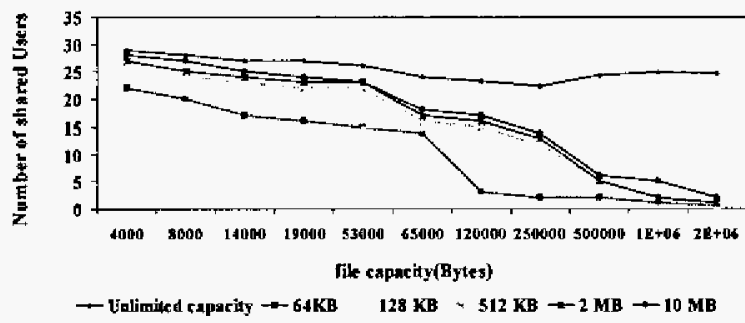

Figure 11: Number of users sharing resources versus file capacity for different capacities of user's buffer.
Figure11 shows the number of users sharing resources versus file capacity for different capacities of user's buffer. For unlimited capacity users it is easily extracted that nearly all users that demand to share a resource file with other users are able to do it in the landscape. On the contrary limited capacity users are liable to search for "empty" users and cache any information in those users. Also for $64 \mathrm{~KB}$ capacity the number of "shared" users drops sensibly.

\section{Conclusions and further research}

In this paper a reliable file sharing scheme for MP2P devices is presented merging the advantages of epidemic file dissemination through fixed and mobile Infostations. The modified epidemic protocol creates a replicated object in order to enable P2P reliable file sharing proving its scalability in nodes" density since it does not require spatial distributions to efficiently spread information while enables reliability in supported mobility.

Next steps in our research are focused in MP2P network's connection with internet via modified Infostations. Additionally the hybridization (Ad-hoc, MP2P-WLANs) of wireless networks is in our research ongoing progress work. The main challenge in wireless multi-hop networks is the efficient routing problem, which is aggravated by node's mobility. Therefore a reliable resource sharing technique for web information retrieval by wireless devices becomes necessity with the tremendous growth of mobile users intending to access the web.

\section{References}

[1] D. J. Goodman, J. Borras, N.B. Mandayam, and R.D. Yates, "INFOSTATIONS: A New System for Data and Messaging Services," Proceedings of IEEE VTC '97 2 (1997) pp.969-973.

[2] S.-Y. Ni, Y.-C. Tseng, Y.-S. Chen, and J.-P. Sheu, "The broadcast storm problem in a mobile ad hoc network". In Proceedings of the Fifth Annual ACM/IEEE International Conference on Mobile Computing and Networking, pages 151-162. ACM Press, 1999.

[3] D. Ganesan, B. Krishnamachari, A. Woo, D. Culler, D. Estrin, and S. Wicker, "Complex behavior at scale: An experimental study of low-power wireless sensor networks". Technical Report UCLA/CSD-TR 02-0013, UCLA, 2002.

[4] A. Demers, D. Greene, C. Hauser, W. Irish, and J. Larson, "Epidemic algorithms for replicated database maintenance". In Proceedings of the Sixth Annual ACM Symposium on Principles of Distributed Computing, pages 1-12. ACM Press, 1987.

[5] J. Kulik, W. R. Heinzelman, and H. Balakrishnan, "Negotiation-based protocols for disseminating information 
in wireless sensor networks". Wireless Networks, 8(23):169-185, 2002.

[6] $\mathrm{X}$. Hong, $\mathrm{K} . \mathrm{Xu}$, and $\mathrm{M}$. Gerla, "Scalable routing protocols for mobile ad hoc networks". IEEE Network Magazine, 16(4):11 -21, Jul-Aug 2002.

[7] Clip2. The Grutella Protocol Specification v0.4, Jun. 2001.

[8] J. Tchakarov and N. Vaidya, "Efficient content location in wireless ad hoc networks". IEEE International Conference on Mobile Data Management (MDM), 2004.

[9] C. D. Gavrilovich, G. Ware, L. Freindenrich, "Broadband Communication of the Higways of Tomorrow", IEEE Communications Magazine, April 2001.

[10] M. Grossglauser and D. Tse, "Mobility Increases the Capacity of Ad Hoc Wireless Networks", Proceedings of IEEE Infocom 2001, pp 312-319.

[11] A. Iacono and C. Rose, "Infostations: New Perspectives on Wireless Data Networks," WINLAB technical document, Rutgers University, 2000.

[12] O. Dousse, P. Thiran, and M. Hasler, "Connectivity in adhoc and hybrid networks," Proceedings of IEEE Infocom 2002, New York, 2002.

[13] T. Small and Z.J. Haas, "The shared Wireless Infostation Model - A New Ad Hoc Networking Paradigm (or Where there is a Whale, there is a Way)".Proceedings of the ACM MobiHoc 2003 conference, Annapolis, Maryland, 2003, pp 233-244.

[14] M. E. J. Newman, "The Movements of North Pacific Blue Whales During the Feeding Season off Southern Califomia and their Southern Fall Migration" Santa Fe Institute 01-12-073.

[15] F. Brauer and C. Ch'avez, "Mathematical Models in Population Biology and Epidemiology" Springer-Verlag New York, Inc., 2001.

[16] T. Camp, J. Boleng, and V. Davies, "A survey of mobility models for ad hoc network research". Wireless Communications \& Mobile Computing (WCMC): Special issue on Mobile Ad Hoc Networking: Research, Trends and Applications, 2(5):483-502, 2002.

[17] K. Park, G. Kim, and M. Crovella, "On the relationship between file sizes, transport protocols and self-similar network traffic". Proceedings of ICNP 1996,pp 171-179.

[18] P. Ulanovs, E. Petersons. "Modeling methods of selfsimilar traffic for network performance evaluation", Scientific Proceedings of RTU. Series 7. Telecommunications and Electronics, 2002.

[19] Z. Haas and M. Pearlman, "The performance of query control schemes for the zone routing protocol". ACM/IEEE Transactions on Networking 9(4), 2001, pp. 427-438.

[20] Z. Haas and M. Pearlman. "The Zone Routing Protocol (ZRP) for Ad Hoc Networks", Internet Draft, draft-ietfmanet-zone-zrp-02.txt, June 1999.

[21] C. Mavromoustakis, H. Karatza, "Adaptive Trafficbased Control Method for Energy Conservation in Wireless Devices". Journal of Simulation Practice and Theory (SIMPRA), Elsevier, accepted for publication (to appear).

[22] C. Mavromoustakis, H. Karatza, "Handling Delay Sensitive Contents using Adaptive Traffic-based Control Method for Minimizing Energy Consumption in Wireless Devices". To appear in the Proceedings of 38th Annual Simulation Symposium (ANSS), IEEE Computer Society Press, SCS, Hilton Mission Valley Hotel, San Diego, CA, April 2-8, 2005.

[23] C. Mavromoustakis, H. Karatza, "Bandwidth Clustering for Reliable and Prioritized Network Routing using Split Agent-based method". To appear in the Proceedings of International Workshop on Assurance in Distributed Systems and Networks (ADSN 2005) in conjunction with the 24th International Conference on Distributed Computing Systems (ICDCS 2005) IEEE Computer Society on Distributed Processing, Columbus, Ohio, USA, June 6, 2005. 